\title{
Moraxella osloensis: an unusual cause of central venous catheter infection in a cancer patient
}

This article was published in the following Dove Press journal:

International Journal of General Medicine

16 October 2012

Number of times this article has been viewed

\section{Yoshiro Hadano' \\ Kenta Ito' \\ Jun Suzuki' \\ Ichiro Kawamura' \\ Hanako Kurai' \\ Kiyofumi Ohkusu² \\ 'Division of Infectious Diseases, Shizuoka Cancer Center, Shizuoka; 2Department of Microbiology, Graduate Medical School of Medicine, Gifu University, Gifu, Japan}

Correspondence: Yoshiro Hadano 2 Otowachinji-cho, Yamashina-ku, Kyoto 607-8062, Japan

Tel +8I75593 4III

Fax +8I7558I 6935

Email hatayoshiyoshi@gmail.com
Abstract: Moraxella osloensis is a rare causative organism of infections in humans, with most cases reported in cancer patients. We report the case of a 67-year-old Japanese man with advanced cancer of the pancreatic head and multiple liver metastases who developed fever with chills. Blood culture was found to be positive for Gram-negative bacilli that were aerobic, oxidase-positive, and catalase-positive. $M$. osloensis was identified by 16 rRNA gene sequencing. Prompt control of the infection was achieved by treatment with cefepime for 14 days, without the need for removal of the central venous catheter.

Keywords: Moraxella osloensis, central venous catheter, infection, cancer

\section{Introduction}

The genus Moraxella consists of aerobic, oxidase-positive, and Gram-negative coccobacilli. Moraxella osloensis has been isolated from environmental sources in hospitals and from the normal human respiratory tract, ${ }^{1}$ and has been reported as a rare causative pathogen of infections in humans. ${ }^{2}$ Although differentiation of $M$. osloensis from other Moraxella species via biochemical testing is cumbersome, accurate identification has recently been achieved using $16 \mathrm{~S}$ ribosomal RNA gene sequencing. ${ }^{3}$ In this paper, we present the first case report of central venous catheter-related infection caused by $M$. osloensis in a Japanese cancer patient, and successful control of the infection without the need for removal of the central venous catheter.

\section{Case report}

A 67-year-old Japanese man with advanced pancreatic head cancer and multiple liver metastases was admitted to our hospital to receive chemotherapy. On day 1, a central venous catheter was inserted and gemcitabine was administered. The clinical course was initially unremarkable, but on day 7 the patient developed fever with chills.

On physical examination, his blood pressure was 106/62 mmHg, pulse was 82 beats per minute, temperature was $38.1^{\circ} \mathrm{C}$, respiratory rate was 24 breaths per minute, and oxygen saturation was $97 \%$ while the patient breathed ambient room air. Physical examination was unremarkable except for mild abdominal distension. Laboratory data obtained on day 9 revealed a white blood cell count of 11,730 per $\mu \mathrm{L}$, with $74 \%$ neutrophils, $11 \%$ lymphocytes, and 10\% monocytes. Hemoglobin was $7.7 \mathrm{mg} / \mathrm{dL}$, with a mean corpuscular volume of $94.9 \mathrm{fL}$, the platelet count was 110,000 per $\mu \mathrm{L}$, and the erythrocyte sedimentation rate was $69 \mathrm{~mm} /$ hour. Serum chemistry revealed a serum sodium of $142 \mathrm{mEq} / \mathrm{L}$, potassium $4.0 \mathrm{mEq} / \mathrm{L}$, chloride $109 \mathrm{mEq} / \mathrm{L}$, blood 
urea nitrogen $19.1 \mathrm{mg} / \mathrm{dL}$, creatinine $0.6 \mathrm{mg} / \mathrm{dL}$, albumin $2.1 \mathrm{~g} / \mathrm{dL}$, total protein $4.7 \mathrm{~g} / \mathrm{dL}$, aspartate transaminase $28 \mathrm{IU} / \mathrm{L}$, alanine transaminase $18 \mathrm{IU} / \mathrm{L}$, total bilirubin $0.6 \mathrm{mg} / \mathrm{dL}$, lactate dehydrogenase $180 \mathrm{IU} / \mathrm{L}$, alkaline phosphatase $404 \mathrm{U} / \mathrm{L}$, amylase $11 \mathrm{U} / \mathrm{L}$, and C-reactive protein $8.1 \mathrm{mg} / \mathrm{dL}$. Urinalysis was normal. A blood culture was obtained, and the patient was treated conservatively because his general condition and vital signs without temperature were stable. On day 9, blood culture of a sample obtained from the central venous catheter was positive for Gramnegative bacilli, so treatment was started with cefepime $1 \mathrm{~g}$ administered intravenously every 8 hours. Peripheral blood culture on day 9 was negative.

On day 10, Moraxella species was isolated from the central venous catheter blood culture, although precise identification was difficult using the BacT/Alert 3D system (Sysmex and BioMérieux, Tokyo, Japan). The organism was oxidase-positive, catalase-positive, and indole-negative. Gram staining of the blood culture revealed two varieties of bacteria, ie, small Gramnegative bacilli and coccobacilli (Figure 1). We performed molecular identification by polymerase chain reaction amplification and sequencing analysis of the 16S rRNA gene using DNA extracted from the isolates. The universal primers, 8UA (5'-AGAGTTTGATCMTGGCTCAG-3') and 1485B (5'-ACGGGCGGTGTGTRC-3'), were used as described previously. ${ }^{4}$ We performed sequencing analysis using a GenBank BLAST search and BiBi (http://pbil.univ-lyon1.fr/) phylogenetic tools. The sequence of the 16S rRNA gene was $99.8 \%$ identical (1442 base pairs over the entire 1445 base pair fragment) to that of $M$. osloensis (ATCC27904, accession number AB643598). Based on this result, we identified the isolate as $M$. osloensis. The isolate exhibited susceptibility to piperacillin, piperacillin/tazobactam, ceftazidime, cefepime, meropenem, gentamicin, levofloxacin,

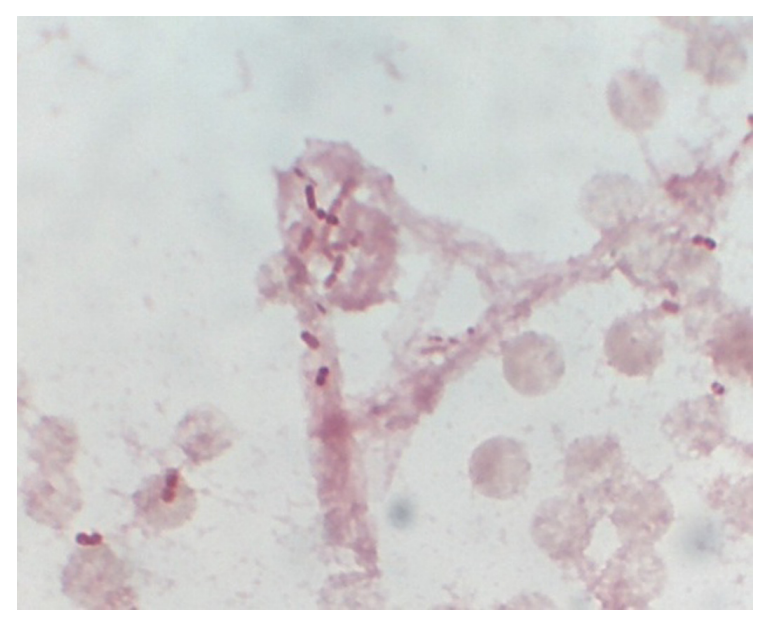

Figure I Gram-staining of blood culture showing Gram-negative small coccobacilli. minocycline, and trimethoprim-sulfamethoxazole. Follow-up blood cultures from the periphery and from the central venous catheter on day 10 were negative and urine culture was negative. The patient underwent a computed tomography scan of the chest and abdomen, with no findings of any new infection.

The patient was continued on cefepime without removal of the central venous catheter because his life expectancy was estimated to be about one month, and he and his family refused to have the central venous catheter removed. On day 13 , significant clinical improvement was noted, so the patient was not treated as a paraneoplastic fever but as suffering from a catheter-related infection due to M. osloensis, The patient was successfully treated with a 2-week course of intravenous cefepime without removal of the central venous catheter. No evidence of relapse of infection was noted, but the patient died as a result of disease progression after 2 months of treatment.

\section{Discussion}

Moraxella species, which are oxidase-positive, nonmotile, and Gram-negative aerobic coccobacilli, are a part of the normal flora of the human skin and mucosal surfaces, as well as inhabitants of the environment. Although Moraxella catarrhalis is the most well known causative organism of respiratory infections such as pneumonia, sinusitis, and otitis media, ${ }^{5}$ the genus Moraxella consists of seven species, including $M$. osloensis.

M. osloensis is a rare causative organism of infections in humans, with most cases reported in immunocompromised hosts, especially cancer patients. ,, $67^{2,7}$ There have been case reports of $M$. osloensis causing bacteremia, ${ }^{2,3}$ central venous catheter-related infection, ${ }^{2,6,8}$ pneumonia, ${ }^{9}$ meningitis, ${ }^{7}$ and endophthalmitis. ${ }^{10}$ The most fluent isolate is from blood samples, as in the present case. ${ }^{11} M$. osloensis is usually considered a Gram-negative bacillus, although in the present case, Gram staining revealed both Gram-negative small bacilli to coccobacilli. This variation in Gram staining pattern and morphology has been reported before. ${ }^{2}$ We could definitively identify the isolate as $M$. osloensis by $16 \mathrm{~S}$ rRNA gene sequencing, which has been reported as a useful method for precise identification. ${ }^{2,3,6,7}$ This method improves clinical microbiological identification by allowing better identification of poorly described organisms like M. osloensis. With respect to treatment, penicillins, cephalosporins, and aminoglycosides are usually effective against $M$. osloensis. ${ }^{11,12}$ In the present case, the patient was successfully treated with a 2-week course of cefepime. Mortality from central venous catheter-related infection caused by $M$. osloensis 
seems to be low. Moreover, in many cases, catheter-related infection caused by this organism resolves without the need for removal of the central venous catheter, as in the present case. ${ }^{2}$ In general, the Infectious Diseases Society of America guideline recommends removal of the catheter in patients with catheter-related bloodstream infection caused by Gram-negative bacilli. ${ }^{13}$ While we also basically recommend removal of a central venous catheter under these circumstances in infections caused by M. osloensis, successful control of the infection may be achieved without removal of the central venous catheter.

In conclusion, we report here a case of central venous catheter-related infection caused by $M$. osloensis and successful control of the infection with antibiotic therapy without removal of the central catheter. To the best of our knowledge, this is the first case of central catheter-related infection caused by $M$. osloensis reported in Japan. The causative organism was identified by $16 \mathrm{~S}$ rRNA gene sequencing. M. osloensis can be a causative pathogen of catheter-related infection among immunocompromised patients, especially in those with underlying cancer, and may be a more important human pathogen than previously thought.

\section{Disclosure}

The authors report no conflicts of interest in this work.

\section{References}

1. Schreckenberger PC, Daneshavar MI, Weyant RS, et al. Acinetobacter, Achromobacter, Chryseobacterium, Moraxella, and other nonfermentative Gram-negative rods. In: Manual of Clinical Microbiology. Washington, DC: American Society for Microbiology; 2003.

2. Han XY, Tarrand JJ. Moraxella osloensis blood and catheter infections during anticancer chemotherapy: clinical and microbiologic studies of 10 cases. Am J Clin Pathol. 2004;121:581-587.
3. Dien Bard J, Lewinski M, Summanen PH, Deville JG. Sepsis with prolonged hypotension due to Moraxella osloensis in a non-immunocompromised child. J Med Microbiol. 2011;60(Pt 1):138-141.

4. Masaki T, Ohkusu K, Hata, et al. Mycobacterium kumamotonense Sp. Nov. recovered from clinical specimen and the first isolation report of Mycobacterium arupense in Japan: novel slowly growing, nonchromogenic clinical isolates related to Mycobacterium terrae complex. Microbiol Immunol. 2006;50:889-897.

5. Murphy TF, Parameswaran GI. Moraxella catarrhalis, a human respiratory tract pathogen. Clin Infect Dis. 2009;49:124-131.

6. Sifri CD, Brassinga AK, Flohr T, et al. Moraxella osloensis bacteremia in a kidney transplant recipient. Transpl Int. 2008;21:1011-1013.

7. Roh KH, Kim CK, Koh E, et al. Three cases of Moraxella osloensis meningitis: a difficult experience in species identification and determination of clinical significance. 2010;25:501-504.

8. Shah SS, Ruth A, Coffin SE. Infection due to Moraxella osloensis: case report and review of the literature. 2000;30:179-181.

9. Vuori-Holopainen E, Salo E, Saxen H, Vaara M, Tarkka E, Peltola H. Clinical "pneumococcal pneumonia" due to Moraxella osloensis: case report and a review. Scand J Infect Dis. 2001;33:625-627.

10. Berrocal AM, Scott IU, Miller D, Flynn HW Jr. Endophthalmitis caused by Moraxella osloensis. Graefes Arch Clin Exp Ophthalmol. 2002;240:329-330.

11. Graham DR, Band JD, Thornsberry C, Hollis DG, Weaver RE. Infections caused by Moraxella, Moraxella urethralis, Moraxellalike groups M-5 and M-6, and Kingella kingae in the United States, 1953-1980. Rev Infect Dis. 1990;12:423-431.

12. Rosenthal SL, Freundlich LF, Gilardi GL, Clodomar FY. In vitro antibiotic sensitivity of Moraxella species. Chemotherapy. 1978;24: 360-363.

13. Mermel LA, Allon M, Bouza E, et al. Clinical practice guidelines for the diagnosis and management of intravascular catheter-related infection: 2009 Update by the Infectious Diseases Society of America. Clin Infect Dis. 2009;49:1-45.
International Journal of General Medicine

\section{Publish your work in this journal}

The International Journal of General Medicine is an international, peer-reviewed open-access journal that focuses on general and internal medicine, pathogenesis, epidemiology, diagnosis, monitoring and treatment protocols. The journal is characterized by the rapid reporting of reviews, original research and clinical studies across all disease areas.

\section{Dovepress}

A key focus is the elucidation of disease processes and management protocols resulting in improved outcomes for the patient. The manuscript management system is completely online and includes a very quick and fair peer-review system. Visit http://www.dovepress.com/ testimonials.php to read real quotes from published authors. 